\title{
IMPORTANCIA DE LA APRECIACIÓN ESTÉTICA EN LA FORMACIÓN DEL MÉDICO
}

\section{Importance of aesthetic appreciation in the training of the doctor}

\author{
Bárbara BARATA CARDOSO루 ; Juana ESTRADA GARZÓN ${ }^{1}$; Elines FRANCO BARATA² \\ 1 Universidad de Ciencias Médicas de La Habana. Facultad de Ciencias Médicas «Comandante Manuel Fajardo» \\ (Cuba). ${ }^{2}$ Empresa Comercializadora de la Música Antonio María Romeu (Cuba).
}

Autor para correspondencia: Barbara Barata Cardoso.

Correo electrónico: barbarabc@infomed.sld.cu

Recibido el 24 de abril de 2020

Aceptado el 8 de mayo de 2020

\section{Resumen}

Introducción: La historia del arte y de la medicina demuestran que el médico y el artista comparten la misma pasión por observar y han colaborado en la construcción de grandes obras científicas, de allí la importancia del uso de las artes visuales en la formación de los estudiantes de medicina.

Objetivo: Promover en los estudiantes de medicina la apreciación del arte en su relación con la profesión médica mediante la vinculación de los conocimientos teóricos y prácticos.

Método: Se realizó un estudio descriptivo, transversal, retrospectivo en el cual se describe la experiencia del curso electivo, "La apreciación estética en su relación con la medicina», el cual es ofrecido a los estudiantes de 5ㅇ año de medicina en el 2018.

Resultados: Participo un total de 55 estudiantes extranjeros. Se realizaron 8 visitas a museos, 6 conferencias ofrecidas por especialistas de arte y patrimonio de la Oficina del Historiador de la Habana. Al ser consultados sobre la importancia y utilidad del curso para su formación como médico, 90,8 \% reconoció su utilidad para su formación profesional.

Conclusiones: La apreciación estética promueve en los estudiantes de medicina la ampliación de la cultura general y el disfrute de las obras de arte, aprovechando la influencia y refinamiento cultural que suele acompañar el mundo del Arte y la Estética, para modelar valores y estimular la sensibilidad en una dinámica de intercambio.

Palabras clave: apreciación estética; estudiante de medicina; arte; medicina. 
IMPORTANCIA DE LA APRECIACIÓN ESTÉTICA EN LA FORMACIÓN DEL MÉDICO

BÁRBARA BARATA CARDOSO; JUANA ESTRADA GARZÓN; ELINES FRANCO BARATA

\section{Summary}

Introduction: The history of art and medicine show that the doctor and the artist share the same passion to observe and have collaborated in the construction of great scientific works, hence the importance of the use of visual arts in the formation of students of Medicine.

Objective: To promote in medical students the appreciation of art in its relationship with the medical profession through the linking of theoretical and practical knowledge.

Method: A descriptive, cross-sectional, retrospective study was carried out in which the experience of the elective course, "The aesthetic appreciation in its relation with medicine», is described, which is offered to the 5th year students of medicine in 2018.

Results: I participate a total of 55 foreign students. There were 8 visits to museums, 6 conferences offered by art and heritage specialists from the Office of the Historian of Havana. When asked about the importance and usefulness of the course for their training as a doctor, $90.8 \%$ recognized its usefulness for their professional training.

Conclusions: The aesthetic appreciation promotes in medical students the broadening of the general culture and the enjoyment of works of art, taking advantage of the influence and cultural refinement that usually accompanies the world of Art and Aesthetics, to model values and stimulate sensitivity in an exchange dynamic.

Keywords: aesthetic appreciation; student of medicine; art; medicine.

\section{Introducción}

La creciente tendencia a la verticalización y especialización de la enseñanza universitaria en el mundo actual ha desestimado la cultura humanística para el desarrollo de la personalidad de los estudiantes, obviando la necesidad de estimular su espiritualidad. Sin embargo, no son pocos los defensores de la concepción de incluir en el currículo cursos sobre artes visuales, literatura y música para garantizar futuros profesionales comprometidos con los valores estéticos y morales más elevados ${ }^{1}$. Como ejemplo del importante intento por rescatar la tradición de incorporar el arte a la medicina debe destacarse el trabajo realizado por el desaparecido Dr. Cs. Néstor Rodríguez Hernández, quien comenzó el proyecto, aún vigente, que vincula el arte y la historia con la Medicina, HISTARMED y que también elaboró con esos temas conferencias, multimedias, programas televisivos y radiales, etc ${ }^{2}$.

Las habilidades de apreciación artística son pobremente desarrolladas en Cuba durante la enseñanza elemental por lo que un elevado porcentaje de los estudiantes de medicina llega a la universidad sin poseerlas ${ }^{3}$.

La historia del arte y de la medicina demuestran que el médico y el artista comparten la misma pasión por observar y han colaborado en la construcción de grandes obras científicas (por ejemplo, la descripción anatómica) y de obras maestras del arte. Así, por ejemplo, Miguel Ángel practicó la disección de cadáveres y estudió pormenorizadamente la forma humana, lo que le permitió esculpir su David, La Virgen y el Niño, y pintar las figuras de la Capilla Sixtina; Leonardo da Vinci realizó frecuentes necropsias en colaboración con el médico Marco Antonio Della Torre, de las que realizaba innumerables dibujos anatómicos de extraordinaria exactitud, y el anatomista Andrea Vesalio tuvo la colaboración de algunos dibujantes discípulos de Tiziano para la realización de la obra De Humani Corporis Fabrica, publicada en 1543, en la que, como se suele decir, se "consumó el más famoso matrimonio entre el arte y la medicina» ${ }^{4}$.

Son numerosos los autores que han resaltado la necesidad de un humanismo médico que complete y complemente la formación de base 


\section{IMPORTANCIA DE LA APRECIACIÓN ESTÉTICA EN LA FORMACIÓN DEL MÉDICO BÁRBARA BARATA CARDOSO; JUANA ESTRADA GARZÓN; ELINES FRANCO BARATA}

científica que preside los planes de estudio de las facultades de medicina, lo cual se podría logran con la motivación del estudiante de medicina hacia la apreciación de la creación artística.

Luego de múltiples observaciones es posible plantear que el trabajo educativo y las acciones de extensión no estimulan en profundidad la apreciación estética y el disfrute del arte como parte de los conocimientos y habilidades específicas en los estudiantes, con lo que se articularían dos procesos centrales de la gestión universitaria, en tal sentido, se describe la experiencia del Curso Electivo denominado, "La apreciación estética en su relación con la medicina», cuyo objetivo es promover en los estudiantes de medicina la apreciación del arte en su relación con la profesión médica mediante la vinculación de los conocimientos teóricos y prácticos. Con lo cual se ha propuesto la inclusión de obras de arte en el proceso enseñanza-aprendizaje para favorecer el rescate de la formación cultural y el gusto por el arte en los estudiantes de medicina.

\section{Método}

Se realizó un estudio descriptivo, transversal, retrospectivo en el que se describe la experiencia del curso electivo «Apreciación estética en su relación con la medicina», en su edición del año 2018, el cual es ofrecido a los estudiantes extranjeros de 5 응 año de medicina en la Facultad de Ciencias Médicas
«Comandante Manuel Fajardo» de La Habana, quienes no reciben la disciplina curricular Filosofía y Sociedad. Este curso electivo consiste en programar visitas didácticas a instituciones de alto valor cultural, tales como, Museo de artes decorativas, Museo Nacional de Bellas Artes, Casa del Vedado, Museo de Farmacia Sarrá, Convento de Belén, la Casa de África, Casa-Museo del Chocolate, Barrio Chino y la Casa de Humboldt; en cuyos lugares los estudiantes pueden intercambiar e interactuar con la creación artística nacional e internacional. Al final de cada edición del curso se realizó una encuesta de satisfacción en la cual cada estudiante describió su experiencia y ofreció recomendaciones, además se desarrolló un taller donde los estudiantes expusieron sobre las obras de artes de sus lugares de origen y la relación con su futura profesión.

En la tabla 1, se describe la distribución por temas del curso según formas de la organización de la enseñanza:

Para el desarrollo de las distintas formas de organización de la enseñanza se emplearon:

Cine - debates.

Simulación educativa.

Juegos, visitas a instituciones de arte y ciencia. Investigaciones científicas de aspectos específicos del contenido.

Conversatorios con especialistas en las diferentes temáticas.

Proyecto Patrimonio.

Tabla 1. Contenidos del curso

\begin{tabular}{|l|c|l|}
\hline \multicolumn{1}{|c|}{ Contenidos } & Tiempo docente & Formas de organización de la enseñanza \\
\hline $\begin{array}{l}\text { 1. Estética y Medicina. Antecedentes. Surgi- } \\
\text { miento y desarrollo. }\end{array}$ & $\mathbf{4 h}$ & Conferencia.Debate audiovisual. \\
\hline 2. Definiciones sobre estética y ciencia. & $\mathbf{6 h}$ & Conferencia.Taller. \\
\hline $\begin{array}{l}\text { 3. La presencia del servicio médico en obras } \\
\text { del arte universal y de Cuba. }\end{array}$ & $\mathbf{6 h}$ & Seminario.Recorrido por museos. \\
\hline $\begin{array}{l}\text { 4. La medicina como arte. Responsabilidad y } \\
\text { desempeño médico. }\end{array}$ & $\mathbf{6 h}$ & Exposición de ponencias. \\
\hline 5. Arte y medicina en la literatura. & $\mathbf{6 h}$ & Trabajo Independiente. \\
\hline Evaluación final. & $\mathbf{4 h}$ & Taller evaluativo final. \\
\hline Total & $\mathbf{3 2 h}$ & \\
\hline
\end{tabular}

Rev. Med. Cine. 2021; 17 (2), 103-109 Ediciones Universidad de Salamanca / అ®@ J. Med. Mov., 2021; 17 (2), $103-109$ 
IMPORTANCIA DE LA APRECIACIÓN ESTÉTICA EN LA FORMACIÓN DEL MÉDICO

BÁRBARA BARATA CARDOSO; JUANA ESTRADA GARZÓN; ELINES FRANCO BARATA

\section{Resultados}

Durante el curso participaron un total de 55 estudiantes extranjeros, de países como Angola, Congo y Sudáfrica (Tabla 2).

Tabla 2. Procedencia de los estudiantes.

\begin{tabular}{|l|c|}
\hline \multicolumn{1}{|c|}{ Nacionalidad } & No de estudiantes \\
\hline Angola & 14 \\
\hline Congo & 19 \\
\hline Sudáfrica & 22 \\
\hline Total & 55 \\
\hline
\end{tabular}

En el curso se realizaron 8 visitas a museos en donde se impartieron 6 conferencias ofrecidas por especialistas de arte y patrimonio de la Oficina del Historiador de la Habana. Entre las actividades colaterales se encuentran la valoración estética de los lugares por donde se transita durante el recorrido hacia las instituciones a visitar.

Al ser consultados sobre la importancia y utilidad del curso para su formación como médico, 90,8 \% reconoció su utilidad para su formación profesional. Así mismo, 94,2 \% manifestó que volvería a participar en actividades similares y 72,1 $\%$ estaría interesado en seguir documentándose en materia de arte de forma

permanente bajo un programa académico oficial.

Cuando se les preguntó sobre qué evento histórico de la salud pública con expresión en la pintura les pareció más interesante, 92,8 \% refirieron que las pinturas relacionadas con las grandes epidemias, una de las opiniones recogidas fue: "me pareció impresionante como recogían a los cadáveres en la vía pública en tiempos de la peste en Europa»; muy posiblemente en alusión a las pinturas, "La plaza del mercado de Nápoles durante la peste de 1656» de Doménico Gargiuloo a la "La peste de Marsella en 1720» de Michel Serre. Y 67,8 \% les pareció interesante y estimulador la pintura, "La lección de anatomía del Dr. Nicolaes Tulp» de Rembrandt, en la cual se muestra una lección de anatomía impartida por el doctor NicolaesTulp a un grupo de cirujanos.

Fue llamativo que 31 estudiantes refirieran que algunas de las expresiones artísticas de su país ofrecidas en algunas de las actividades eran desconocidas por ellos.

\section{Discusión}

No debe extrañar que diferentes autores hayan propuesto el uso de las artes visuales en la formación de los estudiantes de medicina, ni que un grupo creciente de universidades de todo el mundo incluyan cursos de arte en el currículo formativo de los futuros médicos o como actividades optativas o complementarias, ni que el primer departamento de arte médico fuera creado hace más de 100 años por el médico y artista alemán Max Broedel (1870-1941) en la Universidad Johns Hopkins en $1911^{5}$.

Más recientemente, la Universidad de Texas en Galveston imparte cursos de arte desde 1973 en su Instituto de Humanidades Médicas, la Universidad de California hace lo propio desde 1990 y la de Yale desde $2001^{6,7}$.

Se ha estimado que el $65 \%$ de las universidades norteamericanas incluyen actividades de arte en el aprendizaje de la medicina ${ }^{8}$, encontrándose dentro de ellas algunas tan relevantes como Harvard, Columbia, Georgetown, Nueva York, etc., junto con las mencionadas anteriormente ${ }^{9}$. Esta actividad se utiliza también, con los mismos fines, en universidades de Canadá ${ }^{10,11}$, Australia ${ }^{12}$ y en algunas europeas del Reino Unido y Noruega ${ }^{13}$.

Existe abundante información en la literatura educacional acerca de los cursos y estrategias para enseñar arte dentro del currículo de medicina y se mantienen activos programas optativos para enseñar artes a los estudiantes del pregrado y a los residentes de medicina interna en la Universidad Autónoma de Madrid ${ }^{14}$. En la Universidad de Sao Paulo se dictan anualmente dos cursos que tratan las enfermedades infecciosas a través del 
IMPORTANCIA DE LA APRECIACIÓN ESTÉTICA EN LA FORMACIÓN DEL MÉDICO

BÁRBARA BARATA CARDOSO; JUANA ESTRADA GARZÓN; ELINES FRANCO BARATA

arte, otros de posgrado sobre literatura y cine en medicina familiar en la Universidad de Córdoba, Argentina ${ }^{15}$, también de literatura en la Universitat Pompeu Fabra y de cine y literatura en la Autónoma de Barcelona para estudiantes de pregrado y residentes de especialidades médicas ${ }^{16}$. En la Universidad de Pensilvania en EE.UU se dictan sugerentes cursos sobre literatura y medicina reconocidos mundialmente y se publica una revista de humanidades médicas donde pueden leerse las contribuciones literarias de los estudiantes y miembros del Medical College. Esta experiencia se ha extendido a muchas universidades norteamericanas ${ }^{17}$. La Universidad de Salamanca ha desarrollado cursos sobre cine y medicina y una revista especializada "La Revista de Medicina y Cine / Journal of Medicine and Movies», bilingüe, electrónica y de acceso libre ${ }^{18}$.

En Cuba existió una publicación sobre arte dirigida a los médicos llamada "Arte y medicina» que circuló entre los años 1952 y 1959 y que combinaba información artística, histórica, de la ciencia y la cultura en general con artículos científicomédicos ${ }^{19}$, pero cursos curriculares empleando el arte como herramienta pedagógica no se han estructurado hasta el momento ni se emplean regularmente las diversas manifestaciones del arte en el proceso docente-educativo. Como ejemplo de importante intento por rescatar la tradición de incorporar el arte a la Medicina debe destacarse el trabajo realizado con el proyecto que vincula el arte y la historia con la Medicina, HISTARMED².

La mayoría de las actividades se realizan con estudiantes de cursos preclínicos, en grupos reducidos y, mayoritariamente, con la colaboración de especialistas de arte de los museos. También la mayoría de las experiencias reconocen como objetivo principal mejorar el análisis visual mediante una observación estructurada con el fin de que sirva de entrenamiento para la observación clínica, dado su carácter fundamental en la práctica asistencial ${ }^{20}$; pero también se ha señalado que puede favorecer el desarrollo de la empatía, el altruismo o la compasión ${ }^{21}$, la observación de los contextos social, cultural e histórico de la práctica médica, así como el desarrollo personal22.

Hidalgo et al. ${ }^{5}$, reportaron como resultados de un curso sobre la utilidad potencial de las artes visuales en la enseñanza de la medicina, una satisfacción del $78 \%$ de los estudiantes y el hecho de que el $63 \%$ de los participantes consideraron la actividad relevante para su formación.

Una manera fecunda para articular e integrar los procesos de docencia y de extensión universitaria y completar la formación del futuro egresado universitario sería incorporar el arte en el proceso docente-educativo de las asignaturas del currículo de la carrera de medicina, a diferencia de lo que hasta ahora se ha ejecutado en otras partes mediante cursos optativos o curriculares cuyo objeto es el arte y no vinculados particularmente a la impartición de contenidos de las asignaturas. El empleo de imágenes, música y textos de gran belleza y significado, relacionados con las asignaturas durante las actividades docentes, motivaría a los estudiantes y desarrollaría habilidades para la apreciación artística y el disfrute del arte. Además de su inserción en las diversas formas de organización de la enseñanza, los estudiantes realizarían trabajos independientes en forma de ponencias en relación con las obras de arte utilizadas, sus autores, el contexto histórico en que fueron creadas así como para la búsqueda de nuevas obras que pudieran ser incluidas en las clases, con lo que el estudiante ganaría autonomía en la incorporación cultural, pudiendo presentarlas en eventos científicos.

\section{Conclusiones}

La apreciación estética promueve en los estudiantes de medicina la ampliación de la cultura general y el disfrute de las obras de arte, aprovechando la influencia y refinamiento cultural que suele acompañar el mundo del Arte y la Estética, para modelar valores y estimular la sensibilidad en una dinámica de intercambio. 


\section{IMPORTANCIA DE LA APRECIACIÓN ESTÉTICA EN LA FORMACIÓN DEL MÉDICO BÁRBARA BARATA CARDOSO; JUANA ESTRADA GARZÓN; ELINES FRANCO BARATA}

\section{Referencias}

1. Tapajós RA. Introdução das artes nos currículos médicos. Interface (Botucatu) [serial on the Internet]. 2002;6(10):27-36.

2. Betancourt I. Dr. Néstor Rodríguez Hernández. Un hombre de la historia, el arte y la medicina. Rev haban cienc méd 2013;12(3):313-7.

3. Alerm A, González U. Apreciación estética y artística en el proceso enseñanza-aprendizaje de la Medicina. Educ Med Super. 2014;28(4):766-80

4. García M. Medicina y arte. La revolución de la anatomía en el Renacimiento. Rev Cient Soc Esp Enferm Neurol. 2012;35(1):25-27.

5. Hidalgo A, Bordallo J, Cantabrana B. Utilidad potencial de las artes visuales en la enseñanza de la medicina. Rev Educ Med. 2018;19(3):284-93.

6. Haidet $P$, Jarecke J, Adams NE, Stuckey HL, Green MJ, Shapiro D, et al. A frame- work to maximize the power of the arts in medical education: A systematic review. Med Educ. 2016;50(3):320-31.

7. Lake J, Jackson L, Hardman C. A fresh perspective on medical education: The lens of the arts. Med Educ. 2015;49(8):759-72.

8. Rodenhauser P, Strickland MA, Gambala CT. Arts-related activities across U. S. medical schools: A followup study. Teach Learn Med. 2004;16(3):233-9.

9. Wellbery C, Mc Ateer RA. The art of observation: A pedagogical framework. Acad Med. 2015;90(12):162430 .

10. Zazulak J, Halgren C, Tan M, Grierson LE. The impact of an arts-based programme on the affective and congnitive components of empathic development. Med Humanit. 2015;41(1):69-74.

11. Kidd M, Nixon L, Rosenal T, Jackson R, Pereles L, Mitchell $\mathrm{I}$, et al. Using visual art and collaborative reflection to explore medical attitudes toward vulnerable persons. Can Med Educ J. 2016;7(1):22-30.
12. Bramstedt KA. Images healing and learning. The use of visual arts as a window to diagnosing medical pathologies. AMA J Ethics. 2016;18(8):843-54.

13. Frich JC,Fugelli P. Medicine and the arts in the undergraduate me dical curriculum at the University of Oslo Faculty of Medicine, Oslo, Norway. Acad Med. 2003;78(10):1036-8.

14. Barbado FJ. Medicina y literatura en la formación del médico residente de medicina interna. An Med Interna (Madrid) [revista en la Internet].2007;24(4):195-200.

15. Ciuffolini MB, Didoni $M$, Jure $H$, Pinero A. Cine $y$ literatura como elementos para la mediación pedagógica: Una experiencia desde la medicina familiar. Arc. Medic Familiar. 2007;9(3):142-5

16. Loscos J, Baños JE, Loscos F, De la Cámara J. Medicina, cine y literatura: una experiencia docente en la Universidad Autónoma de Barcelona. Rev Med Cine 2006;4:138-42.

17. Baños JE. El valor de la literatura en la formación de los estudiantes de medicina. Educ Med. 2003;6(2):939.

18. González de Dios J. Cine y Pediatría: una oportunidad para la docencia y la humanización en nuestra práctica clínica. Rev Pediatr Aten Primaria. 2010;12(46):299-313.

19. López JA. Índice analítico de la revista Arte y Medicina (1952-1959). ACIMED 2005;13(4).

20. Jasani SK, Saks NS. Utilizing visual art to enhance the clinical observation skills of medical students. Med Teach. 2013;35(7):1327-31.

21. Shapiro J, Rucker L. Can poetry make better doctors? Teaching the humanities and arts to medical students and residents at the University of California, Irvine, College of Medicine. Acad Med. 2003;78(10):953-7.

22. Musso CG, Enz PA. El arte como instrumento educativo en medicina. Arch Argent Pediatr, 2014;112:49295 Arch Argent Pediatr 2014;112(6):494-5. 
IMPORTANCIA DE LA APRECIACIÓN ESTÉTICA EN LA FORMACIÓN DEL MÉDICO

BÁRBARA BARATA CARDOSO; JUANA ESTRADA GARZÓN; ELINES FRANCO BARATA

\begin{tabular}{|l|l|} 
Bárbara Inés Barata Cardoso. Licenciada en Pedagogía. Máster en trabajo \\
Social. Profesora Auxiliar Universidad Médica de La Habana.
\end{tabular}

Rev. Med. Cine. 2021; 17 (2), 103-109 Ediciones Universidad de Salamanca / @@ J. Med. Mov., 2021; 17 (2), $103-109$ [ 109 ] 